\title{
Towards a Probabilistic Understanding About the Context-Dependency of Species Interactions
}

\author{
Chuliang Song, ${ }^{1}$ Sarah Von Ahn, ${ }^{2}$ Rudolf P. Rohr, ${ }^{3}$ and Serguei Saavedra ${ }^{1, *}$
}

Observational and experimental studies have shown that an interaction class between two species (be it mutualistic, competitive, antagonistic, or neutral) may switch to a different class, depending on the biotic and abiotic factors within which species are observed. This complexity arising from the evidence of context-dependencies has underscored a difficulty in establishing a systematic analysis about the extent to which species interactions are expected to switch in nature and experiments. Here, we propose an overarching theoretical framework, by integrating probabilistic and structural approaches, to establish null expectations about switches of interaction classes across environmental contexts. This integration provides a systematic platform upon which it is possible to establish new hypotheses, clear predictions, and quantifiable expectations about the context-dependency of species interactions.

\section{The Context-Dependency of Species Interactions}

Species interactions are central for the persistence (see Glossary) of almost every form of life on Earth [1,2]. However, because biological populations evolve nonlinearly in changing environments [3], species interactions are not fixed and can switch in response to the environmental context established in a given location and time $[4,5]$. Indeed, several studies have shown that the context-dependency of species interactions does not act equally across all classes of interactions [6-9]. That is, an interaction class between two species (be it mutualistic, competitive, antagonistic, or neutral) may switch into a different class at a frequency shaped by the effects of additional interacting species within an ecological community [10] and the abiotic factors within which species are observed [8,9]. For example, in field and laboratory experiments, interactions have been observed to more frequently switch from mutualistic to antagonistic (such as parasitic or predation) than vice versa [7]. Instead, field observations have shown that switches from antagonistic to mutualistic interactions are more frequent in the wild [11,12]. Moreover, these interactions can have different frequencies depending on whether one is focusing on direct interactions, indirect interactions, or higher-order interactions [10,13-16]. This has raised the question of how can we formally and systematically study the context dependency of species interactions $[7,8]$.

However, the complexity arising from the evidence of context-dependencies has underscored a difficulty in establishing a systematic platform to estimate the frequency at which different interaction classes are expected to switch in nature and experiments [8]. In other words, anticipating when the switch of an interaction class will happen and understanding why it will happen are two different questions [17-19]. Indeed, while a lot of work has elucidated the mechanistic principles shaping the context dependency of species interactions [14,20-26], this causative knowledge has done little to increase our predictive power of such interaction switches [27]. In

\section{Highlights}

Species interactions are not fixed and determine the dynamics and persistence of ecological communities.

Empirical work has shown that the switching frequency of species interactions is context-dependent.

The context-dependency of multispecies communities has underscored a difficulty in establishing a quantifiable and systematic framework about the expected frequency of interaction switches.

We propose a structural probabilistic framework to systematically address the uncertainty associated with contextdependency and derive null expectations about the switching frequency of interaction classes in multispecies communities.

${ }^{1}$ Department of Civil and Environmental Engineering, MIT, 77 Massachusetts Av. Cambridge 02139, MA, USA ${ }^{2}$ Department of Mathematics, MIT, 77 Massachusetts Av., Cambridge 02139, MA, USA

${ }^{3}$ Department of Biology - Ecology and Evolution, University of Fribourg Chemin du Musée 10, Fribourg $\mathrm{CH}-1700$, Switzerland

Correspondence:

sersaa@mit.edu (S. Saavedra) 
particular, the difficulty in predicting has resided in how to generalize and transfer the inferred mechanisms beyond specific experiments and study systems [28,29], where both biotic and abiotic factors are hardly the same and fully known [29].

Importantly, interaction switches can strongly affect the dynamics and biodiversity within ecological communities [30]. Thus, in the face of an accelerated rate of climatic variations, answering the question above and knowing which interactions are switching more often than expected is of central importance for developing successful strategies for sustaining ecosystem services that depend on species interactions [5,31-34]. Indeed, understanding and anticipating switches of species interactions has become an important research program in ecological research [35-39]. Yet, because neither the exact equations governing the dynamics of ecological communities nor the full and exact set of biotic and abiotic factors are known, we believe that it is necessary to develop a parallel probabilistic research program to estimate the capacity of species interactions to tolerate or adapt to the changing environmental contexts [10,40-42].

In this line, the structuralist view in biology has provided a systematic and probabilistic way of understanding the diversity (or lack of diversity) that we observe in nature [43-45]. The structural approach posits that ecological communities should have a higher probability of occurrence if their community structures are compatible with a wider range of environmental conditions $[10,46]$. By compatible, we mean the persistence of all the interacting species in a given place and time. Importantly, we can extend this hypothesis to the context dependency of interaction classes. That is, the more an interaction between two species is compatible with community structures and other environmental conditions, the higher its frequency of occurrence. For example, starting with a given community structure (say, two competing yucca moths, Tegeticula intermedia and Tegeticula yuccasella, interacting with a yucca, Yucca filamentosa), there is the possibility that the community is not compatible with the present or future environmental conditions. If this is the case, the community will have to change (say, a switch from pollinator to 'cheater' by one or both moths, [47]). Note that this can happen due to different environmental stressors, distribution ranges, or metabolic properties $[1,47]$. Focusing on the switch of a direct interaction between two species within this initial community, the expected switch will be the one that allows the compatibility between a new community structure and the additional environmental conditions (say, a yucca moth turns into a cheater). Because it is highly likely that these environmental conditions would not be known (i.e., community structures, abiotic factors, initial conditions), following a probabilistic approach we can expect that the realized switch is the one compatible with a wider range of environmental conditions.

Here, we propose estimation of the switching frequencies of interaction classes by using a probabilistic phenomenological approach based on a structuralist view $[10,18,48]$, what we call the structural probabilistic approach. This approach should not be understood as a replacement of mechanistic approaches, but used alongside to provide additional insight into the context dependency of species interactions. In fact, because mechanistic (or deterministic) knowledge is important but difficult to establish and parameterize, our proposed approach focuses on the phenomenological direct effects between species, which can be easier to estimate in practice [49-52]. Specifically, we propose the sources of context dependency are disentangled into two probabilistic measures: (i) the possibility of transition from one community structure to a different one, what we call the probability of transition; and (ii) the possibility of persistence of species within the new community structure, what we call the probability of persistence. Following the structuralist view [43], these two probabilistic measures are treated as being independent. The transition probability is related to biophysical constraints imposed within species interactions

\section{Glossary}

Community structure: the quantitative description of the phenomenological direct effects between the biological populations within a studied community. This is typically known as the interaction matrix. Note that the inverse of this matrix provides the phenomenological total (direct and indirect) effects between populations.

Constrained parameter space: the subset of the full parameter space with only ecological possible parameters under ecological constraints. Context-dependency of species interactions: the fact that the sign and magnitude of species interactions can change as a function of the environmental conditions. Direct interaction: the mechanistic effect of one population on the growth rate of another population (e.g., the mutualistic direct effect between a tree and the seed disperser), without considering the mediating effects from other populations and resources (e.g., the second tree and geographical space). Dynamical stability: the property of a system to tolerate small perturbations on population abundances without changing into a quantitatively different state over the long term.

Ecological community: the set of interacting biological populations (e.g., species or individuals) in a given place and time (e.g., two trees and one seed disperser). What constituted an ecological community is relative to the resolution of a study. For example, if the dynamics of the soil microbiome acting on trees will be explicitly studied, then this microbiome should be part of the ecological community. Otherwise, it can be implicitly integrated into the dynamics of trees (e.g., via the intrinsic growth rates or interactions).

Environmental conditions: abiotic factors and any biotic factors that are not explicitly included in the community structure.

Feasibility: the existence of an equilibrium point at which all species have positive abundance. Note that this is a prerequisite for persistence. Full parameter space: the entire parameter space with all possible parameter values.

Higher-order interaction: the aggregated mechanistic effect of two or more populations on the growth rate of a population after the removal of direct and indirect effects. 
and determined the set of possible (or impossible) changes in a system. The probability of persistence is related to the extent to which arbitrary or constrained changes in environmental conditions affect the persistence of species within a given community structure [53]. That is, once a transition is selected, the probability of persistence determines whether this new community structure can be sustained or not. Overall, we propose a systematic platform to establish null expectations about switches of interaction classes across context-dependent multispecies dynamics.

\section{The Structural Probabilistic Approach}

In ecology, the structuralist view has been adapted to study the persistence of species within a given community structure under changing environments [10,40,46,54,55]. This structural approach integrates the notion of structural stability into population dynamics models [10]. Conceptually, a dynamical system is structurally stable if a small change in the system parameters does not change its qualitative behavior [56-59]. However, because of the uncertainties associated with the exact equations and external perturbations governing the dynamics of ecological systems, the structural approach in ecology has extended the notion of structural stability from a dichotomous to a quantitative definition. That is, instead of only answering whether a system is structurally stable or not, the structural approach answers by how much the system is structurally stable. This extension has allowed us to quantify, for a dynamical system, the range of conditions (parameter values) compatible with a particular qualitative behavior (e.g., stable coexistence).

For example, the structural approach has quantified the structural stability of the persistence of species within a given community structure $[46,53,60]$. This structure is summarized by the interaction matrix [49], where each element is the phenomenological direct effect of one species on the growth rate of another species. Note that the inverse of this interaction matrix (a.k.a total-effects matrix) corresponds to the total phenomenological effect (direct an indirect) of one species on another (i.e., how the abundance of the species is expected to change due to a perturbation on the abundance of another species $[61,62])$. Thus, the structural stability of the persistence of species is given by the set of phenomenological parameter values (assuming a population dynamics model) compatible with a given community structure. In the classic Lotka-Volterra (LV) model, these parameters are called the intrinsic growth rates, which represent the summary of biotic and abiotic effects (not explicitly considered in the community) acting upon species $[63,64]$. Thus, the set of parameter values compatible with the persistence of species is typically called the domain of persistence of the community structure $[10,65]$. The larger the domain of persistence, the larger the structural stability of the community structure. Importantly, structural stability is a useful indicator for both short $[66,67]$ and long time-scale ecological dynamics $[60,68,69]$

While the structural approach provides a systematic methodology to investigate the behavior of multispecies communities under changing environments, it also provides a probabilistic interpretation. For instance, suppose we do not have any a priori knowledge about how the intrinsic growth rates will be changing within a community, then assuming a probabilistic approach, our best naive guess is that all parameter values are equally likely in the entire parameter space (Box 1). Then, the probability of persistence of species within a given community structure can be estimated by looking at the size of the domain of persistence relative to the full parameter space (Box 1). Importantly, if there is some a priori knowledge about the range of possible parameter values, the structural approach also allows us to take into account this conditional probability by measuring the size of the domain of persistence relative to a constrained parameter space [53] (Box 1).
Indirect interaction: the mechanistic effect of one population on the growth rate of another population through a mediating set of populations and resources (e.g., the effect between the trees when competing for the seed disperser and soil nutrients). Interaction class: the sign of the effect of one population on the growth rate of another population.

Parameter space: the set of parameter values of a dynamical system.

Persistence: the property of a system to sustain positive abundances for all its constituent populations across time. Feasibility and dynamical stability provide the necessary and sufficient conditions for persistence. To take into account cyclic populations, persistence can be defined in terms of permanence. Permanence conditions for three-species communities are: the three-species community has to be feasible, the determinant of the 3D matrix has to be positive, and the determinants of the three $2 \mathrm{D}$ submatrices have to be positive [97].

Phenomenological direct effect: the summary of direct and indirect (mechanistic) effects of one population on the growth rate of another population (e.g., the sum of exploitative competition effects between the two trees), without considering the mediating effects from other populations within the studied community (removal of the mediating effect of the seed disperser, but leaving the effect of nutrients).

Phenomenological indirect interaction: the summary of phenomenological direct effects of one population on the growth rate of another population through a mediating set of populations considered within a studied community (e.g., the effect of apparent competition between trees caused by the seed disperser), without considering the phenomenological direct effect between the two populations (e.g. removal of the effect of other populations and nutrients).

Phenomenological intrinsic growth rates: the summary effect between mortality rate and resource intake (abiotic and biotic resources not considered explicitly in the community structure).

Probability of persistence: the possibility that a population persists within a given community structure. 


\section{The Switching Probability of Interaction Classes}

To illustrate the structural probabilistic approach, let us consider again a simple community formed by three species (e.g., two yeasts, Metschnikowia gruessii and Starmerella bombicola, and one nectar flower, Mimulus aurantiacus), $[26,70])$, whose community structure is summarized by the interaction matrix $\mathbf{A}$. The elements $a_{i j}$ denote the phenomenological direct effect of species $j$ on species $i$ [49] and such effects can be classified as: mutualistic $(+,+)$, antagonistic $(+,-)$, competitive $(-,-)$, neutral $(0,0)$, amensalistic $(-, 0)$, and commensalistic $(+, 0)$. Note that $a_{i i}$ corresponds to the phenomenological effect of a species on itself.

To systematically investigate switches from one interaction class to another class between two species (e.g., the two yeast), we need to consider point transitions between interaction classes. In general, these transitions can be of any number of steps. However, due to biophysical constraints, experimental and observational studies [7,26,71] have shown that these are typically one-point transitions [e.g., transitions from competitive to antagonistic $(-,-) \rightarrow(+,-)$, from mutu-

\section{Box 1. Probability of Persistence \\ Following previous work on structural stability in ecological research $[10,46,53]$, it is possible to calculate the persistence probability $\Omega(\mathbf{A})$ of a given community structure $\mathbf{A}[53]$. Persistence is guaranteed by the existence of positive (feasible) and stable equilibrium abundances of the system as a function of model parameters [97]. Note that feasibility is the neces- sary condition for persistence regardless of the specific definitions of persistence [97]. In other words, all criteria of persis- tence should be added upon feasibility. For example, to deal with persistent cyclic or chaotic populations that are not dynamically stable, we can add permanence upon feasibility as the criteria for persistence $[97,98]$.}

Assuming that ecological dynamics can be described by any model topologically equivalent to the Lotka-Volterra population dynamics model [49], it has been proved that the domain of feasibility $D_{\digamma}(\mathbf{A})$ is described by a convex cone with the edges defined by the columns of the interaction matrix $\mathbf{A}$ [73] (Figure I). This cone is made of vectors, the elements of which are phenomenological intrinsic growth rate values leading to feasible solutions. Then, to obtain persistence, one has to restrict the domain of feasibility to the stable and feasible abundances (although this definition can change as mentioned above). That is, one has to determine the domain of stability $D_{S}(\mathbf{A})$. Note that $D_{S}(\mathbf{A})$ is necessarily contained inside $D_{\digamma}(\mathbf{A})$, as stability is defined only for feasible equilibria. Therefore, the intersection of the domains of feasibility and stability can be called the domain of persistence $D_{P}(\mathbf{A})=D_{F}(\mathbf{A}) \cap D_{S}(\mathbf{A})$ (Figure I). The larger $D_{P}(\mathbf{A})$, the larger the structural stability of persistence of a community with community structure $\mathbf{A}$. Consequently, following a probabilistic approach, the probability of persistence $\Omega(\mathbf{A})$ is given by the proportion of $D_{P}(\mathbf{A})$ within the parameter space of phenomenological intrinsic growth rates [10,53]:

$\Omega(\mathbf{A})=$ proportion of $D_{P}(\mathbf{A})$ in the parameter space.

Formally speaking, $\Omega(\mathbf{A})$ is the normalized solid angle of the convex cone of persistence $D_{P}(\mathbf{A})[10,53]$. Finally, one can constrain the parameter space of phenomenological intrinsic growth rates to a domain $\mathcal{C}$ (e.g., considering only positive or negative intrinsic growth rates) and define the conditional probability of persistence $\Omega(\mathbf{A} \mid \mathcal{C})$ :

$\Omega(\mathbf{A} \mid \mathcal{C})=$ proportion of $D_{P}(\mathbf{A})$ inside $\mathcal{C}$,

which is computed as the fraction (conditional probability)

$\Omega(\mathbf{A} \mid \mathcal{C})=\frac{\Omega(\mathbf{A} \cap \mathcal{C})}{\Omega(\mathcal{C})}$

Finally, the average probability of persistence of a randomly chosen species within the community structure $\mathbf{A}$ (assuming independence) is given by

$\omega(\mathbf{A})=\Omega(\mathbf{A})^{1 / n}$

where $n$ is the number of species in the community. The computation of all these quantities can be done following the guidelines of Song et al. [53]. Further information and the code to compute these probabilities are provided as supplemental information (see Supplemental Information online).

\section{Probability of transition: the} possibility that two populations can switch between two interaction classes. Structural approach: a quantitative extension of the notion of structural stability. Specifically, this approach is concerned with the extent to which a model can be perturbed before changing into a qualitatively different behavior. Note that this qualitative behavior is defined by the research question (e.g., persistence, feasibility, permanence, dynamical stability, chaos, etc.).

Structural stability: traditionally, structural stability is the capacity of a dynamical system to tolerate small perturbations to the vector field without qualitatively changing its phase portrait. In other words, the capacity of a dynamical system to tolerate small perturbations in the model itself without changing to a qualitatively different behavior. That is, a system is structurally stable or not.

Switching probability: the probability that a switch from one interaction class to another class happens between two populations.

Total-effects matrix: the summary of phenomenological direct and indirect effects of how perturbing the abundance of a species affects the abundance of another species. Mathematically, it is defined as the inverse of the community structure. 


\section{(A) Without constraints}

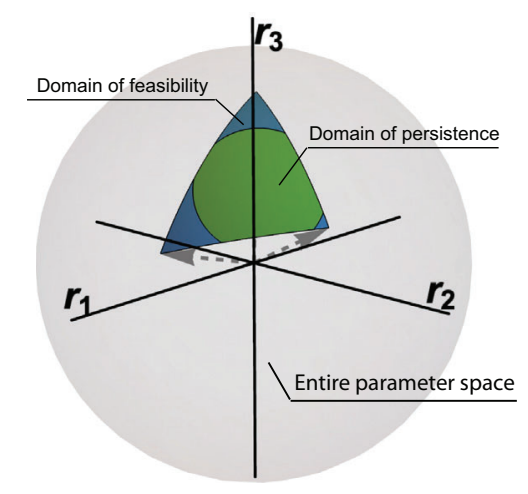

(B) With constraints

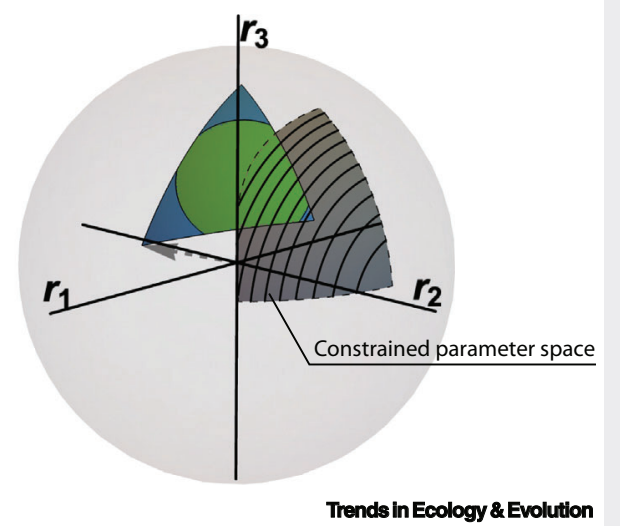

Figure I. Probability of Persistence. The figure shows two examples of the probability of persistence of a threespecies community characterized by a community structure $\mathbf{A}$ under dynamics topologically equivalent to the LotkaVolterra model. The light gray region (unit sphere) represents the entire parameter space of phenomenological intrinsic growth rates governing the dynamics of the community. The blue and green regions correspond to the domains of feasibility $D_{\digamma}(\mathbf{A})$ and persistence $D_{P}(\mathbf{A})$, respectively. The broken arrows are the spanning vectors of the domains of feasibility. Panel (A) shows that without constraints (or prior information) in the parameter space, the probability of persistence is equivalent to the fraction of the domain of persistence inside the sphere. Instead, panel (B) shows that under abiotic constraints, the parameter space reduces to a constrained region (dark gray region). In this case, the probability of persistence becomes the fraction of the domain of persistence inside the constrained parameter space.

alistic to antagonistic $(+,+) \rightarrow(+,-)$, from antagonistic to competitive $(+,-) \rightarrow(-,-)$, from antagonistic to mutualistic $(+,-) \rightarrow(+,+)$, and from neutral to commensalistic $(0,0) \rightarrow(+, 0)$, etc.] Thus, two species can switch their interaction class if it is possible to move from an initial community structure $\mathbf{A}$ to a community structure $\mathbf{B}$ by a given point transition (e.g., a mutualistic interaction between two yeasts can switch into an antagonistic interaction [26]). Note that there can be one or more interaction switches within the community (e.g., yeast can switch from competitive to amensalistic, while a yeast-flower interaction can switch from mutualism to parasitism). The difference between two community structures (interaction matrices) will be the sign (and potentially the magnitude) of one or more interactions ( $a_{i j}$ and $\left.a_{j i}\right)$. That is, moving from the community structure $\mathbf{A}$ to $\mathbf{B}$ implies at least a switch of an interaction class between two species. Note that the magnitude of the intraspecific interactions $\left(a_{i i}\right)$ can also change. Once an interaction has switched and a new community structure has emerged, the population dynamics, in combination with the environmental conditions (summarized by the intrinsic growth rates in the LV model), will determine whether such community structure can persist. As we mentioned before, persistence may have short or long time-scales [11]; however, both can be captured by structural stability $[60,66-69]$.

We propose to estimate the switching probability of interaction classes by calculating the joint probability that : (i) community structure A (e.g., two competing trees, Pistacia lentiscus and Quercus ilex, and a seed disperser with both trees, Sylvia melanocephala, [72]) transitions to community structure $\mathbf{B}$ (e.g., two competing trees, a seed disperser with the first tree and seed predator with the second tree); and (ii) the species can persist within the new community structure B. From a probabilistic point of view, the first probability corresponds to the ratio of favorable transitions to the number of possible transitions. This implies that this first probability measure is conditioned on the initial community structure and the expected point transitions 
Box 2. Switching Probability of Interaction Classes

The switching probability from one interaction class to another (i.e., from community structure $\mathbf{A}$ to community structure B) is defined as $p_{\mathbf{A} \rightarrow \mathbf{B} \mid \mathcal{C}}$ and can be estimated by the product of two probability measures: (i) the probability of transition from a given community structure $\mathbf{A}$ to a new community structure $\mathbf{B}$; and (ii) the probability of persistence $\omega(\mathbf{B} \mid \mathcal{C})$ of the new community structure $\mathbf{B}$ under some environmental context $\mathcal{C}$ (Box 1). That is, we can write

$p_{\mathbf{A} \rightarrow \mathbf{B} \mid \mathcal{C}}=$ probability of transition $(\mathbf{A} \rightarrow \mathbf{B}) \cdot \omega(\mathbf{B} \mid \mathcal{C})$.

In the absence of information, as a null expectation, all potential transitions can have equal chance of happening (Figure I). Mathematically, this probability can be expressed as

probability of transition $(\mathbf{A} \rightarrow \mathbf{B})=1 / L_{\mathrm{A}}$,

where $L_{A}$ corresponds to the number of potential transitions from the initial biotic structure $\mathbf{A}$ (Figure l). This null expectation can be improved with more biological information, such as forbidden links and mutation rates [99].

\section{(A) Multiple potential transitions}

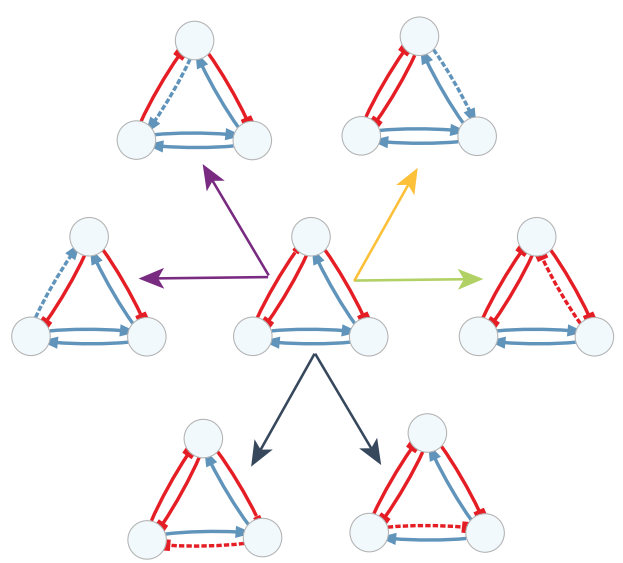

(B) Single potential transition

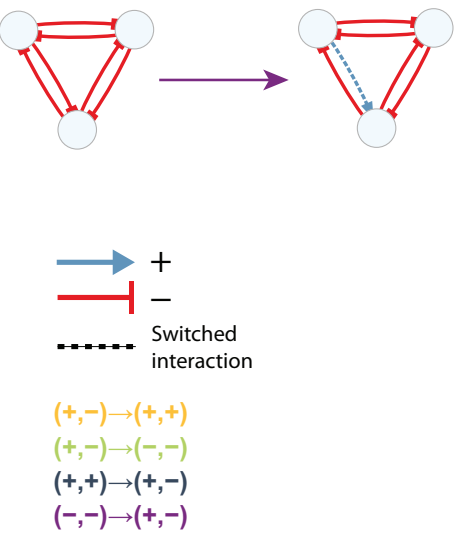

Trends in Ecology \& Evolution

Figure I. Probability of Transition. For illustration purposes, the figure shows two examples of the probability of transition of interaction classes when assuming one-point transitions and one transition at a time. Each node represents a species. The blue, red, and broken links represent positive, negative, and switched interactions, respectively. The color of links represent the different types of one-point transitions that are possible starting from a three-species community structure. Panel (A) shows that a three-species community with three different interaction classes (one mutualistic, one competitive, and one antagonistic) yields six possible transitions. Panel (B) shows that a three-species community with only one interaction class (all competitive) yields one possible transition. While this example is for onepoint transitions, the structural probabilistic approach can accommodate other types of transitions. For example, switching from a competitive interaction to a mutualistic interaction (two-point transition).

(Box 2). For example, by considering one switch at a time over one-point transitions, a threespecies community with competitive interactions (e.g., three competing trees) has only one possible transition, while a community with a mixture of interaction classes has several possible transitions (Box 2). Similarly, the second probability measure is given by the ratio of favorable parameter values (environmental conditions) compatible with community structure $\mathbf{B}$ to the number of possible parameter values. This second probability measure is conditioned by the assumed governing dynamics (e.g., intrinsic growth rates in the LV model) and the range of parameters values under consideration (Box 3). Under the structural approach, the probability of persistence is efficiently calculated using Monte Carlo techniques for the family of models topologically equivalent to LV dynamics, even for a large number of species $[53,73]$. Thus, the joint probability of transition and persistence provides a null frequency 
that can be used to investigate which interaction classes are more likely to switch under different environmental contexts.

\section{Probability Diagrams to Study Context-Dependency}

The application of the structural probabilistic approach can be leveraged by building probability diagrams describing the relative frequency of interaction switches within a given community. The nodes of the diagrams correspond to the community structures $(\mathbf{A}, \mathbf{B}, \mathbf{C}, \ldots)$, while the arrows represent the switching probabilities from one community structure to a different one. For example, let us consider community structures representing the combination of interaction classes among three species. Additionally, let us consider one-point transitions, only one transition at a time within the community, and four interaction classes: mutualistic $(+,+)$, antagonistic $(+,-)$, competitive $(-,-)$, and neutral $(0,0)$. Note that this is equivalent to studying interaction switches under rapid dynamics within ecological building blocks [74-78].

Following the assumptions above, Figure 1 shows a graphical illustration of the probability diagram describing different sign combinations (community structures) and switches of interaction classes within a three-species community. Any of the nodes (triangles) can be treated as the initial community structure. Note that the number of potential transitions varies depending on the

Box 3. Switching Probability Under Environmental Contexts

It has been shown that the switching frequency of interaction classes depends on the environmental context experienced by species [7-9]. For instance, it has been observed that mutualism is the most likely to switch in field or laboratory experiments [7], whereas mutualism is the least likely to switch in the wild [8]. These contrasting patterns can be systematically studied and explained by estimating the switching probability from mutualism to antagonism $p_{\mathbf{M} \rightarrow \mathbf{A} \mid \mathcal{C}}$ and vice $\operatorname{versa} p_{\mathbf{A} \rightarrow \mathbf{M} \mid \mathcal{C}}$ following the structural probabilistic approach.

For example, consider one community with two possible community structures $\mathbf{M}$ and $\mathbf{A}$, which are the same except for one interaction between the same pair of species: mutualistic in $\mathbf{M}$ (e.g., a tree and a seed disperser) while antagonisticin $\mathbf{A}$ (e.g., a tree and a seed predator). As a first-order of approximation, we can assume the probability of transition inversely related to the number of possible transitions from the initial community structures $\left(L_{\mathbf{M}}\right.$ and $\left.L_{\mathbf{A}}\right)$. Additionally, without prior information, we can consider an unconstrained parameter space of phenomenological intrinsic growth rates (i.e., $\mathcal{C}=$ none) for a community in the wild. Instead, for experiments, we can assume that the parameter space is constrained by the predetermined initial community structure (i.e., $\mathcal{C}=\mathbf{A}$ for the switch $\mathbf{A} \rightarrow \mathbf{M}$, whereas $\mathcal{C}=\mathbf{M}$ for the switch $\mathbf{M} \rightarrow \mathbf{A}$, Figure I). This implies that we have to compare the following probabilities for each environmental context:

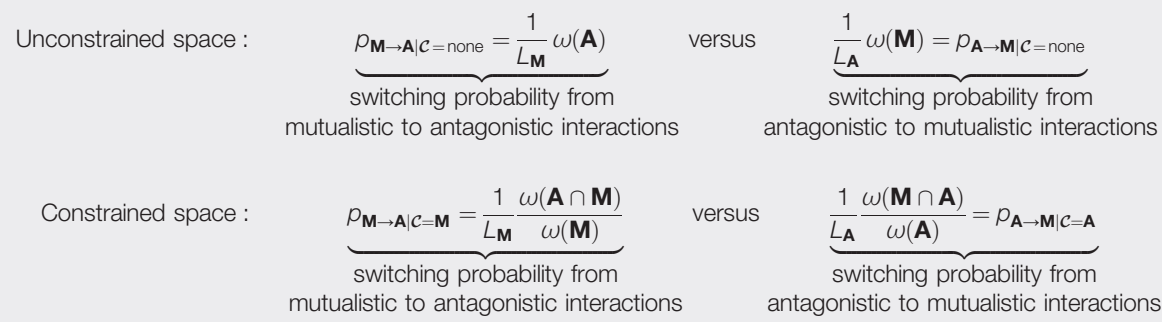

Importantly, this null model can be applied for all combinations of interaction classes. Figurel provides a numerical example, where it is shown that the expected switch is almost twice as large from antagonism to mutualism under the unconstrained parameter space, but this pattern reverses under a constrained parameter space. Moreover, it is possible to see that regardless of the switching direction, the frequency can vary depending on the precise initial community structure. These qualitative results are the same when we use permanence instead of dynamical stability as a criterion for persistence (see the supplemental information online). Overall, this example reveals that a naive phenomenological probabilistic approach rooted on the structuralist view can already explain the results reported in laboratory and field experiments [7,8]. Moreover, while we have focused on three-species communities, our approach can be scaled to larger dimensions as the computation of these metrics is relatively inexpensive (code provided in the online supplemental information). 


\section{(A) Without constraints}

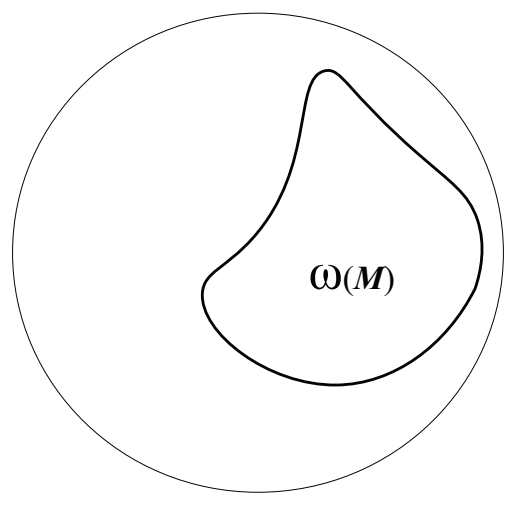

Probablity of persistence $=\omega(M)$

\section{(C) Without constraints}

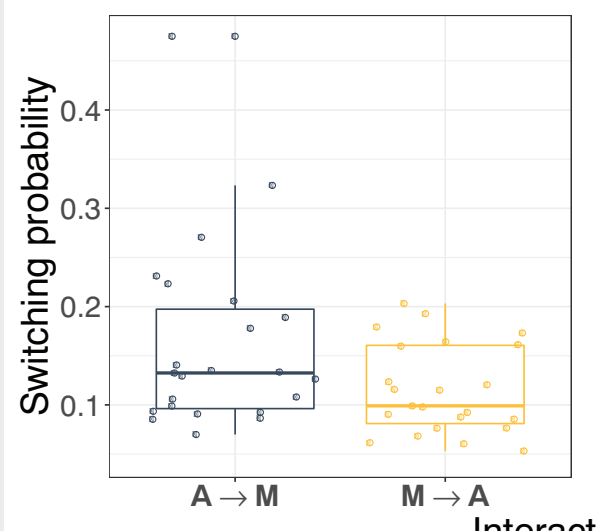

\section{(B) With constraints}

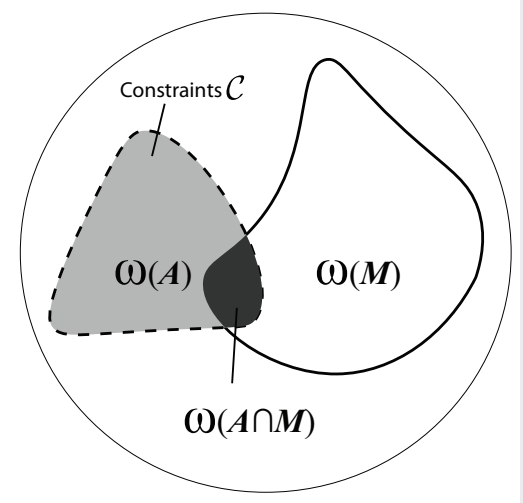

Probablity of persistence $=\frac{\omega(A \cap M)}{\omega(A)}$

(D) With constraints

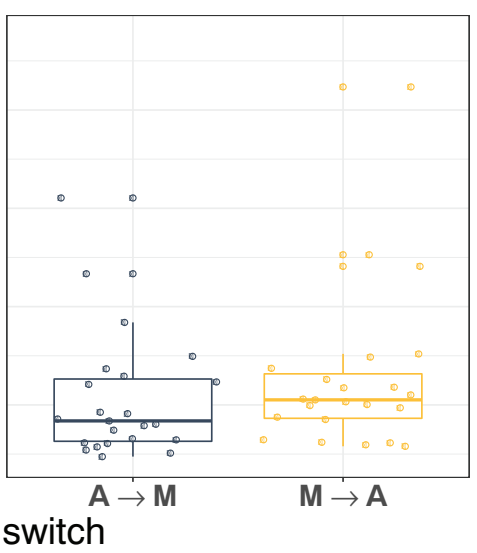

Trends in Ecology \& Evolution

Figure I. Switching Probability Under Different Environmental Contexts. Panels (A) and (B) show a graphical example of how constraints acting on the parameter space (environmental conditions) can change the probability of persistence and, as a consequence, the switching probability from a community structure $\mathbf{A}$ to a community structure $\mathbf{M}$. Note that this methodology can be applied in the opposite direction (i.e., from community structure $\mathbf{M}$ to $\mathbf{A}$ ). Panel (A) considers the case where no constraint acts on the persistence of the community structure $\mathbf{M}$ [i.e., $\omega(\mathbf{M} \mid \mathcal{C}=$ none)]. That is, all parameter values can occur with equal likelihood inside the parameter space (represented as the circle), implying that the persistence of $\mathbf{M}$ only depends on its proportion inside the circle. Panel (B) considers the case where the parameter space is constrained by the domain of persistence of the initial community structure $\mathbf{A}$. Under this constrained space, community structure $\mathbf{M}$ can persist with probability $\omega(\mathbf{M} \mid \mathcal{C}=\mathbf{A})=\frac{\omega(\mathbf{A} \cap \mathbf{M})}{\omega(\mathbf{A})}$. Panels $(C)$ and $(D)$ show a numerical example of the estimated switching probability with and without constraints (as shown in Panels $A$ and B). Legends $\mathbf{A} \rightarrow \mathbf{M}$ and $\mathbf{M} \rightarrow \mathbf{A}$ denote switches from antagonistic to mutualistic interactions and vice versa, respectively. Each point corresponds to the switching probability (estimated as in Box 3) for a given initial three-species community structure. Note that these initial community structures need to have the interaction class given by the switch. All initial community structures have unique combinations of interaction classes within each boxplot. The community structures are parameterized following a half normal distribution with mean zero and variance one for the nondiagonals (signs are predetermined based on the interactions classes), and set to -1 for all diagonal elements (assuming self-regulation). We assume one-point transitions, one transition at a time, and the transition probability given by the inverse of the number of potential transitions. The switching probability is the mean of 1000 ensembles. Box plots show the mean and interquartile range. Note that the expected switching probability from all initial community structures is higher for $\mathbf{A} \rightarrow \mathbf{M}$ without constraints (as it has been reported in the wild) and lower for $\mathbf{A} \rightarrow \mathbf{M}$ with constraints (as it has been reported in experiments). The scattered points are the result of the heterogeneity given by the differences of biotic contexts (initial community structures). The code to generate these probabilities is provided in the online supplemental information. 


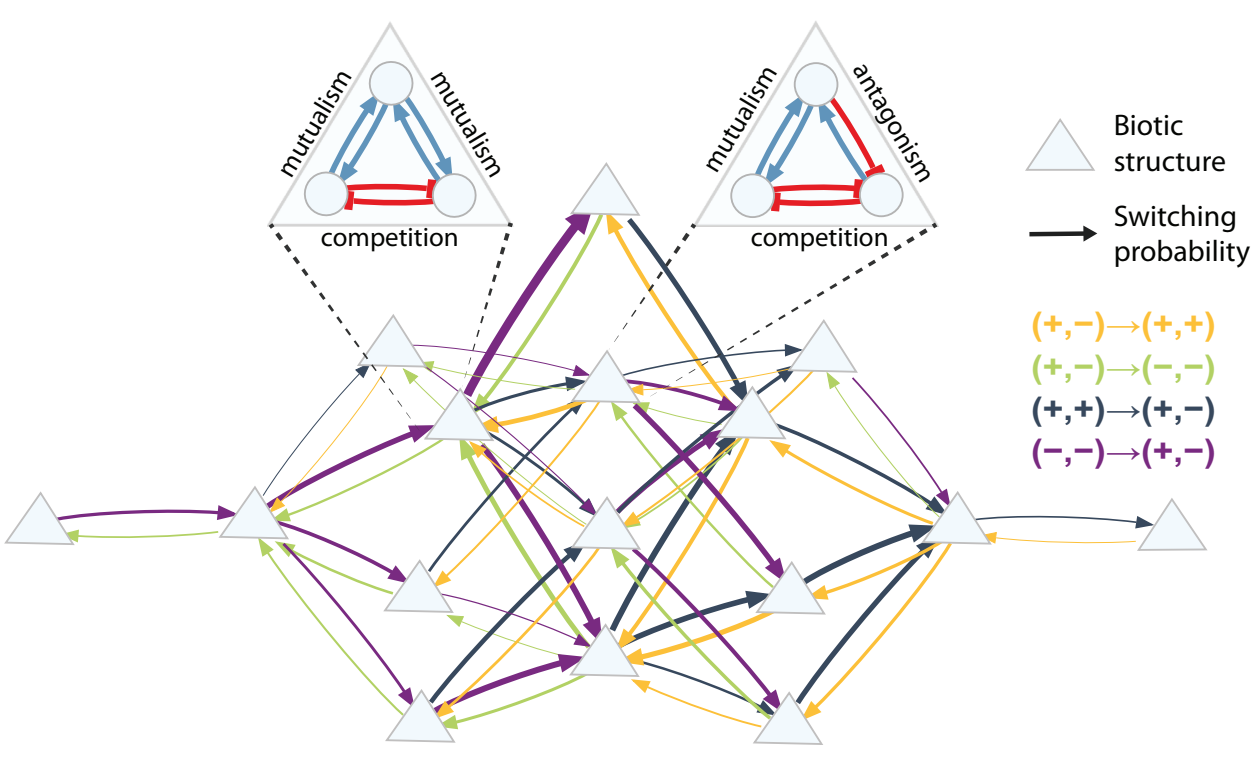

Trends in Ecology \& Evolution

Figure 1. Probability Diagram to Study the Context-Dependency of Species Interactions. To illustrate the structural probabilistic approach, the figure shows a probability diagram for a given three-species community assuming onepoint transitions of interaction classes and one transition at a time. Each node (triangle) represents a different community structure (characterized by a given combination of interaction classes) that can describe the three-species community. For example, the node in the zoomed node in the left describes a community structure $\mathbf{A}$ formed by tree 1 competing with tree 2 $(-,-)$, while animal 3 is acting as a seed disperser with both trees $(+,+)$. In turn, the node in the right describes a community structure $\mathbf{B}$ formed by the same two trees competing with each other, but this time animal 3 is acting as a seed predator with tree $1(-,+)$ and as a seed disperser with tree $2(+,+)$. Hence, there are directed (although thin) links between nodes $\mathbf{A}$ and $\mathbf{B}$ given that community structure $\mathbf{A}$ can switch (with low probability) into community structure $\mathbf{B}$ (and vice versa) via a one-point transition (Box 2). The thickness of each link is proportional to the switching probability from one structure to another (Boxes 2 and 3). The direction and color of each link represent the direction and type of switch: purple $(-,-) \rightarrow(+,-)$, green $(+,-) \rightarrow(-,-)$, yellow $(+,-) \rightarrow(+,+)$, and blue $(+,+) \rightarrow(+,-)$. For visualization purposes, this diagram only represents a subset of potential interaction switches for a three-species community. Note that it is only necessary to locate the initial community structure (can be any node) and follow the probability diagram to study the expected switches of interaction classes.

starting community structure. The color of the arrows represents a specific switch from an interaction class into another interaction class; whereas the thickness represents the expected frequency (i.e., the switching probability). Importantly, these probability diagrams can be extended to incorporate more species, transitions, and interaction classes.

Therefore, using probability diagrams, it is possible to differentiate the expected frequency of interaction switches, or even track the most probable initial community structures from which an observed community structure may have originated. Furthermore, by changing the parameter space (i.e., environmental conditions), the probability diagrams will change the thickness of links, representing the updated context dependency of species interactions. For example, theoretically it has been shown that mutualistic interactions on average enlarge the domain of persistence $[46,66,79,80]$. That is, community structures with mutualistic interactions will increase their probability of persistence, leading to a higher compatibility with different environmental contexts. This implies that by allowing the full parameter space to represent the set of environmental contexts makes the links pointing towards communities with mutualistic interactions thicker than average (Box 3). This relationship is also consistent with the idea that mutualism enlarges species' realized niche [81] or geographic range [82], allowing mutualistic interactions to be present in different environmental contexts. However, this also implies that if the parameter space is constrained by an initial community structure containing mutualistic interactions, it is likely that 
this structure will share a higher than average fraction of environmental contexts with other community structures and eventually switch into a different interaction class (Box 3). Thus, depending on the assumed environmental context, the switching probability can be higher from mutualistic to antagonistic interactions or vice versa (Box 3). Yet, these idiosyncratic responses can be systematically studied using probability diagrams (Figure 1).

\section{Generating Probability Diagrams: A Brief Guideline}

To generate probability diagrams, we provide the following brief guideline. First, we need to define the number of species forming the community structure (i.e., the nodes). Note that in the complete absence of information, it is possible to generate three-species communities, where the 'third' species can simply represent the phenomenological summary effects of unknown species on the two species under consideration. Second, we need to define the nature of species interactions within a community structure. That is, to specify any forbidden type of interaction classes and the phenomenological direct effects between species. These direct effects can be point estimators, range of values, or distributions (e.g., half normal distribution). If the knowledge of the direct effects is a range or a distribution, the analysis should be made over an ensemble of community structures. Third, we need to establish the links between the nodes in the diagram. This is done by assuming (using prior knowledge) the possible point transitions between community structures. Note that depending on the nature of transitions, it can be possible or not to introduce multiple changes when moving to a new community structure.

Next, we estimate the weights of the links in the probability diagram, corresponding to the switching probability between two community structures. The value of these links is the product of the probability of transition and the probability of persistence. The probability of transition takes into account the information about mutation and transition rates. That is, this probability should be motivated by any phenomenological understanding about the system under investigation. In the absence of information, this probability can be simply assumed as the inverse of the number of possible transitions coming out of a community structure (Box 2). In turn, the estimation of the probability of persistence requires the phenomenological knowledge about the range of environmental conditions compatible with the new community structure. We propose this probability is calculated following the structural approach (Box 1). That is, the range should be measured relative to the parameter space. Importantly, both the range and the parameter space can be constrained. For example, the parameter space can be constrained by trophic positions, metabolic properties, or the initial community structures [53]. Similarly, the range of parameter values can be shaped by an underlying distribution. In the absence of information, environmental conditions can be phenomenologically modeled using the full parameter space under a uniform distribution (Box 3). In sum, these steps provide a well-defined null model to test whether interaction classes are switching more or less than expected under a certain set of assumptions or environmental contexts. Finally, one can verify theoretical frequencies with experimental and observational data (example provided in Box 3) $[67,68]$.

\section{Concluding Remarks}

Recent meta-analyses on context dependency of species interactions have shown that mutualism is most likely to switch to antagonism across contexts, while antagonism is least likely to switch to mutualism [7]. Yet, the effect size of these empirical results has not been particularly strong, reflecting that the switching frequency also depends on how researchers measure context dependency $[8,9]$. Similarly, field studies have shown that the importance of mutualistic interactions relative to competitive interactions depends strongly on abiotic factors, becoming more important under extreme conditions, also known as the stress gradient hypothesis (SGH) [11]. Indeed, over the course of evolutionary history, mutualisms have tended to persist and are

\section{Outstanding Questions}

How can we establish a formal and systematic framework to know whether interactions are switching more or less often than expected by chance?

How can we integrate the complexity dimensionality, and uncertainty associated with switches of species interactions in multispecies communities?

How can we develop an alternative approach to use alongside a mechanistic understanding to explain and anticipate switches of species interactions in multispecies communities?

Is it possible to build a probabilistic understanding to study the context dependency of species interactions in multispecies communities?

Can two species have a larger probability to become mutualistic rather than competitive because of the environmental context?

How can we reconcile the observations that mutualisms have tended to persist in nature despite being the most likely to switch in experiments? 
present in almost every ecosystem [1]. Take the symbiosis of plants and mycorrhizal fungi as an example: the ancestor of all seed plants associated with mycorrhizae over 400 million years ago and most seed plants still do so today [83]. However, as mentioned before, experimental work has suggested that this highly persistent interaction class is the most likely to switch [7]. Thus, how can we reconcile the observations and establish a formal framework when these mutualisms have tended to persist in nature despite being the most likely to switch in experiments (see Outstanding Questions)?

Traditionally, these questions have been looked at through mechanistic approaches; however, the context dependency of species interactions makes it unclear whether we can generalize the causal knowledge from specific experimental settings [28,29,84]. Indeed, even if we have a complete map of all the causal factors affecting switches of species interactions, getting all the necessary data would be unfeasible in practice: it is virtually impossible to know the exact equations governing the dynamics of species interactions, the exact parameter values, or how and when external perturbations are going to modify both equations and parameters, especially when studying multispecies dynamics. One possibility would be to focus only on qualitative frameworks such as SGH [11,12]. Indeed, SGH has provided a rich conceptual road map to theoretically and empirically investigate interaction switches across environmental gradients. However, the contrasting community responses, due to the diversity of biotic and abiotic factors, have revealed that assumptions related to SGH need to be relaxed or added, depending on the environmental contexts [85-88]. This has raised a need for a systematic platform to include the large number of present species, the vast heterogeneity of community structures, and substantially large combinations of environmental conditions that can eventually lead to establish general conclusions about interaction switches [89,90].

To tackle the problem of context dependency, we have proposed integration of phenomenological and probabilistic approaches under a structuralist view. This integration is not intended to substitute empirical work or mechanistic approaches, but to provide a parallel theoretical framework that generates hypotheses, clear predictions, and quantifiable expectations following a systematic methodology. In particular, we have proposed separation of the switching probability of interaction classes into two quantifiable measures: the probability of transition and the probability of persistence. Those probabilities are derived from a phenomenological perspective, thus any measured information of environmental contexts can reduce the uncertainty of the estimation for a multispecies community. Importantly, both probability measures are only limited by the established assumptions and current advances of structural stability in ecology. For example, currently, the probability of persistence is limited to dynamical systems topologically equivalent to LV dynamics. That is, systems with several alternative equilibria [91], higher-order interactions [92,93], coevolution [94], or spatial structures [95,96] are not currently considered. However, these limitations can be addressed by future work. It is also worth noting that the flexibility of the approach imposed by phenomenological parameters is also a limitation for its scope. Specifically, while this approach can provide a null frequency about context dependency, it cannot provide in itself causative knowledge. Yet, this can be improved by integrating mechanistic process into the probability measures in order to define more realistic point transitions and parameter spaces. Similarly, our methodology can be useful to develop an inverse approach to the context dependency problem: inferring the transition probabilities given observed community structures. Overall, our proposed approach should be taken as a parallel first-order approximation to the problem of context dependency of species interactions. Yet, we hope that this framework can help to move the field of ecology towards a formal probabilistic thinking, especially for multispecies and context-dependent dynamics. 


\section{Acknowledgments}

We thank Mohammad AlAdwani, Judith Bronstein, Haoran Cai, Simone Cenci, Lucas Medeiros, and Pengjuan Zu for insightful comments. We also thank our editor and three anonymous reviewers for their highly constructive comments that improved the quality of the manuscript. S.S. acknowledges funding by the Mitsui Chair. R.P.R. acknowledges funding from the Swiss National Science Foundation, Grant Number 31003A_182386.

\section{Supplemental Information}

Supplementary information associated with this article can be found online at https://doi.org/10.1016/j.tree.2019.12.011.

\section{References}

1. Thompson, J.N. (2005) The Geographic Mosaic of Coevolution, Chicago University Press

2. Grant, P.R. and Grant, B.R. (2014) 40 Years of Evolution. Darwin's Finches on Daphne Major Island, Princeton University Press

3. Sugihara, G. (1994) Nonlinear Forecasting for the Classification of Natural Time Series. Philos. Trans. Royal Soc. A 348, $477-495$

4. Levins, R. (1968) Evolution in Changing Environments: Some Theoretical Explorations, Princeton University Press

5. Odum, E.P. and Barrett, G.W. (2005) Fundamentals of Ecology (fifth edition), Thomson, Brooks-Cole

6. Bronstein, J.L. (1994) Conditional outcomes in mutualistic interactions. Trends Ecol. Evol. 9, 214-217

7. Chamberlain, S.A. et al. (2014) How context dependent are species interactions? Ecol. Lett. 17, 881-890

8. Frederickson, M.E. (2017) Mutualisms are not on the verge of breakdown. Trends Ecol. Evol. 32, 727-734

9. Maestre, F.T. et al. (2005) Is the change of plant-plant interactions with abiotic stress predictable? Ameta-analysis of field results in arid environments. Funct. Ecol. 93, 748-757

10. Saavedra, S. et al. (2017) A structural approach for understanding multispecies coexistence. Ecol. Monogr. 87, 470-486

11. Callaway, R.M. (1998) Competition and facilitation on elevation gradients in subalpine forests of the northem Rocky Mountains, USA. Oikos 82, 561-573

12. Callaway, R.M. et al. (2002) Positive interactions among alpine plants increase with stress. Nature 417, 844-848

13. Abrams, P.A. (1987) On classifying interactions between populations. Oecologia 73, 272-281

14. Levine, J.M. et al. (2017) Beyond pairwise mechanisms of species coexistence in complex communities. Nature 546, 56-64

15. AlAdwani, M. and Saavedra, S. (2019) Is the addition of higher-order interactions in ecological models increasing our understanding of ecological dynamics? Math. Biosci. 35, 108222

16. Terry, J.C.D. et al. (2017) Trophic interaction modifications: an empirical and theoretical framework. Eco. Lett. 20, 1219-1230

17. Breiman, L. et al. (2001) Statistical modeling: The two cultures. Stat. Sci. 16, 199-231

18. May, R.M. (2004) Uses and abuses of mathematics in biology. Science 303, 790-793

19. Song, C. et al. (2019) On the consequences of the interdependence of stabilizing and equalizing mechanisms. Am. Nat. 194 $627-639$

20. Moeller, H.V. and Neubert, M.G. (2016) Multiple Friends with Benefits: An Optimal Mutualist Management Strategy? Am. Nat. 187, E1-E2

21. Koffel, T. et al. (2018) Facilitation-vs. competition-driven succession: the key role of resource-ratio. Ecol. Lett. 21, 1010-1021

22. Palmer, T.M. et al. (2008) Breakdown of an ant-plant mutualism follows the loss of large herbivores from an African savanna. Science 319, 192-195

23. Nathaniel Holland, J. and DeAngelis, D.L. (2009) Consumerresource theory predicts dynamic transitions between outcomes of interspecific interactions. Ecol. Lett. 12, 1357-1366

24. Hillesland, K.L. and Stahl, D.A. (2010) Rapid evolution of stability and productivity at the origin of a microbial mutualism. Proc. Nat1. Acad. Sci. 107, 2124-2129

25. Rakoff-Nahoum, S. et al. (2016) The evolution of cooperation within the gut microbiota. Nature 533, 255
26. Hoek, T.A. et al. (2016) Resource availability modulates the cooperative and competitive nature of a microbial crossfeeding mutualism. PLOS Biol. 14, e1002540

27. Shalev-Shwartz, S. and Ben-David, S. (2014) Understanding machine learning: From theory to algorithms, Cambridge University Press

28. Lawton, J.H. (1999) Are there general laws in ecology? Oikos 84 177-192

29. Currie, D.J. (2019) Where Newton might have taken ecology. Glob. Ecol. Biogeogr. 28, 18-27

30. Vellend, M. (2016) The Theory of Ecological communities (MPB-57), Princeton University Press

31. Dirzo, R. et al. (2014) Defaunation in the Anthropocene. Science $345,401-406$

32. Tylianakis, J.M. et al. (2008) Global change and species interactions in terrestrial ecosystems. Ecol. Lett. 11, 1351-1363

33. Scheffers, B.R. et al. (2016) The broad footprint of climate change from genes to biomes to people. Science 354,719

34. Alexander, J.M. et al. (2015) Novel competitors shape species' responses to climate change. Nature 525, 515-518

35. Thébault, E. and Fontaine, C. (2010) Stability of ecological communities and the architecture of mutualistic and trophic networks. Science 329, 853-856

36. Mougi, A. and Kondoh, M. (2012) Diversity of interaction types and ecological community stability. Science 337, 349-351

37. Coyte, K.Z. et al. (2015) The ecology of the microbiome: Networks, competition, and stability. Science 350, 663-666

38. Barbier, M. et al. (2018) Generic assembly patterns in complex ecological communities. Proc. Natl. Acad. Sci. 115, 2156-2161

39. Yan, C. and Zhang, Z. (2019) Impacts of consumer-resource interaction transitions on persistence and long-term interaction outcomes of random ecological networks. Oikos 128, 1147-1157

40. Cenci, S. et al (2018) Rethinking the importance of the structure of ecological networks under an environment-dependent framework. Ecol. Evol. 8, 6852-6859

41. Cenci, S. and Saavedra, S. (2019) Non-parametric estimation of the structural stability of non-equilibrium community dynamics. Nat. Ecol. Evol. 3, 912

42. Tóth, A.B. et al. (2019) Reorganization of surviving mammal communities after the end-Pleistocene megafaunal extinction. Science 365, 1305-1308

43. Alberch, P. (1989) The logic of monsters: Evidence for internal constraint in development and evolution. Geobios 22, $21-57$

44. Gould, S.J. (2002) The Structure of Evolutionary Theory, Harvard University Press

45. Valverde, S. et al. (2018) The architecture of mutualistic networks as an evolutionary spandrel. Nat. Ecol. Evol. 2, 94

46. Rohr, R.P. et al. (2014) On the structural stability of mutualistic systems. Science 345, 1253497

47. Pellmyr, O. and Leebens-Mack, J. (2000) Reversal of Mutualism as a Mechanism for Adaptive Radiation in Yucca Moths. Am. Nat. 156, S62-S76

48. Justus, J. (2008) Ecological and Lyapunov Stability. Philos. Sci. $75,421-436$

49. Case, T.J. (2000) An Illustrated Guide to Theoretical Ecology, Oxford University Press, Oxford

50. Cenci, S, et al, (2019) Regularized S-map for inference and forecasting with noisy ecological time series. Methods Ecol. Evol. 10, 650-660 
51. Pomeranz, J.P. et al. (2019) Inferring predator-prey interactions in food webs. Methods Ecol. Evol. 10, 356-367

52. Gibson, T.E. et al. (2016) On the origins and control of community types in the human microbiome. PLOS Comput. Biol. 12, e1004688

53. Song, C. et al. (2018) A guideline to study the feasibility domain of multi-trophic and changing ecological communities. J. Theor. Biol. 450, 30-36

54. Saavedra, S. et al. (2014) How structurally stable are global socioeconomic systems? J. Royal Soc Interface 11, 20140693

55. Godoy, O (2019) Coexistence theory as a tool to understand biological invasions in species interaction networks: Implications for the study of novel ecosystems. Funct. Ecol. 33, 1190-1201

56. Prigogine, I. (1962) Introduction to nonequilibrium statistical mechanics, Interscience Press

57. Andronov, A. and Pontriagin, L. (1937) Systémes Grossiers. Dokl. Akad. Nauk 247-251

58. Smale, S. (1967) Differentiable Dynamical Systems. Bull. Amer. Math. Soc. 73, 747-817

59. Strogatz, S.H. (2014) Nonlinear dynamics and chaos: with applications to physics, biology, chemistry, and engineering, Westview Press

60. Song, C. et al. (2018) Structural changes within trophic levels are constrained by within-family assembly rules at lower trophic levels. Eco. Lett. 21, 1221-1228

61. Dambacher, J.M. et al. (2002) Relevance of community structure in assessing indeterminacy of ecological predictions. Ecology $83,1372-1385$

62. Novak, M. et al. (2016) Characterizing species interactions to understand press perturbations: what is the community matrix? Annu. Rev. Ecol. Evol. Syst. 47, 409-432

63. Svirezhev, Y.M. and Logofet, D.O. (1983) Stability of Biological Communities, Mir Publishers

64. Cadotte, M.W. and Tucker, C.M. (2017) Should Environmental Filtering be Abandoned? Trends Ecol. Evol. 32, 429-437

65. Logofet, D.O. (1993) Matrices and Graphs: Stability Problems in Mathematical Ecology, CRC Press

66. Saavedra, S. et al. (2016) Nested species interactions promote feasibility over stability during the assembly of a pollinator community. Ecol. Evol. 6, 997-1007

67. Song, C. and Saavedra, S. (2018) Structural stability as a consistent predictor of phenological events. Proc. Royal Soc. B 285, 20180767

68. Saavedra, S. et al. (2017) Reorganization of interaction networks modulates the persistence of species in late successional stages. J. Ani Ecol. 86, 1136-1146

69. Cenci, S. et al. (2018) Estimating the effect of the reorganization of interactions on the adaptability of species to changing environments. J. Theor. Biol. 437, 115-125

70. Letten, A.D. et al. (2018) Species coexistence through simultaneous fluctuation-dependent mechanisms. Proc. Nat. Acad. Sci. 6745-6750

71. Johnson, N.C. et al. (1997) Functioning of mycorrhizal associations along the mutualism-parasitism continuum. New Phytol. 135, 575-586

72. González-Varo, J.P. et al. (2019) The timing of frugivoremediated seed dispersal effectiveness. Mol. Ecol. 28, 219-231

73. Cenci, S. and Saavedra, S. (2018) Structural stability of nonlinear population dynamics. Phys. Rev. E 97, 012401

74. Pimm, S.L. (1982) Food webs. In Food webs, pp. 1-11, Springer

75. Holt, R. and Hochberg, M. (2001) Indirect interactions, community modules and biological control: a theoretical perspective. In
Evaluating indirect ecological effects of biological control, pp. 13-37

76. Solé, R.V. and Bascompte, J. (2005) Self-Organization in Complex Ecosystems (MPB-42), Princeton University Press

77. Milo, R. et al. (2002) Network motifs: simple building blocks of complex networks. Science 298, 824-827

78. McCann, K.S. (2011) Food webs (MPB-50), Princeton University Press

79. Song, C. and Saavedra, S. (2018) Will a small randomlyassembled community be feasible and stable? Ecology 99, 743-751

80. Song, C. et al. (2017) Why are some plant-pollinator networks more nested than others? J. Animal Ecol. 86 (6), $1417-1424$

81. Bruno, J.F. et al. (2003) Inclusion of facilitation into ecological theory. Trends Ecol. Evol. 18, 119-125

82. Afkhami, M.E. et al. (2014) Mutualist-mediated effects on species' range limits across large geographic scales. Ecol. Lett. 17, 1265-1273

83. Maherali, H. et al. (2016) Mutualism persistence and abandonment during the evolution of the mycorrhizal symbiosis. Am. Nat. 188, E113-E125

84. Fukami, T. (2018) Messy Communities: The Arising Researcher. Bull. Ecol. Soc. Am. 99, 58-59

85. Holmgren, M. and Scheffer, M. (2010) Strong facilitation in mild environments: the stress gradient hypothesis revisited. J. Ecol. $98,1269-1275$

86. Maestre, F.T. et al. (2009) Refining the stress-gradient hypothesis for competition and facilitation in plant communities. J. Ecol. 97, 199-205

87. Malkinson, D. and Tielborger, K. (2010) What does the stressgradient hypothesis predict? Resolving the discrepancies. Oikos 119, 1546-1552

88. Ie Roux, P.C. and McGeoch, M.A. (2010) Interaction intensity and importance along two stress gradients: adding shape to the stress-gradient hypothesis. Oecologia 161, 733-745

89. Barrio, I.C. et al. (2013) Extending the stress-gradient hypothesis - is competition among animals less common in harsh environments? Oikos 122, 516-523

90. Michalet, R. et al. (2014) Interaction intensity and importance along two stress gradients: adding shape to the stressgradient hypothesis. J. Veg. Sci. 25, 609-613

91. Bunin, G. (2017) Ecological communities with LotkaVolterradynamics. Phys. Rev. E 95, 042414

92. Letten, A.D. and Stouffer, D.B. (2019) The mechanistic basis for higher-order interactions and non-additivity in competitive communities. Eco. Lett. 22, 423-436

93. AlAdwani, M. and Saavedra, S. (2019) Is the addition of higherorder interactions in ecological models increasing the understanding of ecological dynamics? Math. Biosci. 315, 108222

94. Medeiros, L.P. et al. (2018) The geographic mosaic of coevolution in mutualistic networks. Proc. Nat. Acad. Sci. 115, 12017-12022

95. Leibold, M.A. and Chase, J.M. (2017) Metacommunity ecology, vol. 59. Princeton University Press

96. Guzman, L.M. et al. (2019) Towards a multi-trophic extension of metacommunity ecology. Eco. Lett. 22, 19-33

97. Hofbauer, J. and Sigmund, K. (1998) Evolutionary Games and Population Dynamics, Cambridge University Press

98. Benaïm, M. and Schreiber, S.J. (2019) Persistence and extinction for stochastic ecological models with internal and external variables. J. Math. Biol. 79, 393-431

99. Olesen, J.M. et al. (2010) Missing and forbidden links in mutualistic networks. Proc. Royal Soc. B 278, 725-732 